\title{
RESEARCH
}

\section{PSYCHOLOGICAL COMPLAINTS, TREATMENT COMPLIANCE, AND ONLINE CONSULTATION EXPECTATIONS OF GERIATRIC PSYCHIATRIC PATIENTS IN COVID-19 PANDEMIC: A CROSS- SECTIONAL EVALUATION}

Turkish Journal of Geriatrics
DOI: $10.31086 /$ tigeri.2021.247

2021; 24(4): 499-509

- Hasan KAYA ${ }^{1}$

- Aybeniz CIVAN KAHVE ${ }^{1}$

- Merve ELTEMIZ1

- Ezgi CELLAT ${ }^{1}$

- Erol GÖKA ${ }^{1}$

CORRESPONDANCE

${ }^{1}$ Hasan KAYA

University of Health Sciences Ankara City Hospital, Department of Psychiatry, ANKARA Turkey

Phone: +905054925449

e-mail: dr.kaya.hasan@gmail.com

Received: Jun 29, 2021

Accepted: Nov 20, 2021

${ }^{1}$ University of Health Sciences Ankara City Hospital, Department of Psychiatry, ANKARA, Turkey

\section{Abstract}

Background: This study aimed to determine how the psychological complaints of geriatric patients with mental disorders were affected by the COVID-19 pandemic and how they continued their treatment during this period. In addition, this work sought to determine the social support resources of these patients and their expectations regarding online consultations.

Methods: Telephone interviews were carried out with 776 patients over the age of 65 who had a mental disorder. A sociodemographic data form, the Patient Health Questionnaire-9, the General Anxiety Disorder-7 were employed to evaluate their psychological complaints.

Results: A total of 733 patients (94.5\%) were unable to go to outpatient visits, although 607 (78.2\%) patients used their medications regularly. The most important factor contributing to continue drug treatment was the extension of drug reports by the government. There was an increase in psychiatric complaints in $318(41.0 \%)$ patients. Anxiety (23.7\%), insomnia (17.0\%), and depression (13.3\%) were the most common complaints. $72.7 \%$ of the patients wanted to have an online consultation with their psychiatrist. Predictors for depression were increased age and female gender. Predictors for anxiety were disease duration of five years or more and irregular medication intake during the pandemic.

Conclusion: The high-risk groups in terms of increasing psychiatric complaints must be identified for psychosocial interventions. During the COVID-19 pandemic, government policies on the health system played an important role in ensuring continued treatment for geriatric psychiatric patients. It is also important to be able to conduct online psychiatric consultations during extraordinary situations.

Keywords: COVID-19; Mental Disorder; Aged; Depression; Anxiety. 


\section{INTRODUCTION}

Coronavirus disease 2019 (COVID-19), which is caused by severe acute respiratory syndrome coronavirus 2 (SARS-CoV-2), has seriously affected public health globally. In mild cases, coughing, sore throat, or fever are experienced, whereas severe cases may result in pneumonia, acute respiratory distress syndrome, multiple-organ failure, and even death.

Several risk factors, such as advanced age, suppressed immune system, preexisting respiratory and cardiovascular diseases, and low social support, have been found to contribute to severe disease symptoms (1). Conditions that occur with aging, such as lung anatomy changes, decreased respiratory reserve, and deterioration in the immune system's functions, may cause the disease to worsen in older adults with COVID-19 (2).

During this period, a campaign was launched with the slogan "Stay at Home," wherein people were advised not to go out unless they had to carry out essential activities in Turkey. On one hand, many news articles and content related to COVID-19 are now being shared on television and across social media platforms throughout the world. News coverage related to COVID-19 often features mortality and morbidity in older adults. Unfortunately, the reporting and sharing of scary images from intensive care units has increased fear among the elderly population (3). Indeed, the COVID-19 pandemic has psychologically affected an increasing number of people, and studies have shown the prevalence of generalized anxiety disorder, depression, insomnia, and post-traumatic stress disorder symptoms in many people (4).

Some people may be considered psychosocially at risk during the pandemic. For example, the elderly and individuals with other medical diseases, such as immune system disorders, may be physically and psychologically at risk from the impacts of COVID-19. It has also been reported that healthcare professionals caring for COVID-19 patients, those with a previous psychiatric illness, or those diag- nosed with alcohol or substance use disorder are more psychologically affected than other members of society $(5,6)$.

Governments around the world have imposed different restrictions on the population at risk. For example, in Turkey, a curfew was implemented for people aged 65 and above on March 21, 2020. With these restrictions and changes in health services, it is an important question how people over the age of 65 will continue their treatment and how they will be affected psychologically.

This study aimed to determine how the psychological complaints of geriatric patients with mental disorders were affected by the COVID-19 pandemic and how they continued with their treatment. In addition, this work sought to determine the social support resources of these patients and their expectations about online consultations.

\section{MATERIALS AND METHODS}

\section{Ethical considerations}

The study was approved by the University of Health Sciences Ankara City Hospital Ethics Committee (No. E1-20-717) and was conducted according to the criteria set by the Helsinki declaration. All participants provided verbal informed consent after fully understanding the benefits and risks of participation.

\section{Procedure}

Patients aged 65 and above who were admitted to the Ankara City Hospital Psychiatry Outpatient Clinic between September 1, 2019 and March 1, 2020 and scored three or less on the Clinical Global Impression-Severity Scale were included in the study. The diagnosis made by the psychiatrist who followed up on each patient with a clinical interview and entered the system was also accepted as valid.

The phone numbers of the patients were accessed through the hospital registration system. 
Information about the study was provided to each patient during telephone interview, a sociodemographic data form and study scales were administered after obtaining their verbal consent. Two experienced psychiatrists made all the phone calls, which lasted 20-30 minutes each. If the person could not be reached from the called number, two more attempts at two different times were made. Individuals who could not be reached after at least three calls at three different times were excluded from the study.

\section{Measurement tools}

\section{Sociodemographic data form}

This form was prepared by the researchers. It includes the sociodemographic information of the participants. In addition, questions containing information about how patients continue the follow-up and treatment process for mental disorders are also included in the form. They were also asked about their regular medication use during the interviews. Patients who stated that they continued the recommended medication regularly until the interview date at the final outpatient visit were recorded as regular medication users.

\section{Patient Health Questionnaire-9 (PHO-9)}

The PHQ-9 is a nine-item self-reported scale designed to screen for depression. The items are graded using a 4-point Likert-type scale ranging from 0 (not at all) to 3 (almost daily). Total scores range from 0-27 and are classified as follows: 0-4: "minimal or no," 5-9: "mild," 10-14: "moderate," 15-19: "moderate-severe," and 20-27 as "severe." (7) The reliability and validity of this scale on a Turkish sample was verified by Sarı et al. (8).

\section{General Anxiety Disorder-7 (GAD-7)}

The GAD-7 is a seven-item, self-reported questionnaire designed to screen for anxiety. The items here are graded on a 4-point Likert-type scale ranging from 0 (not at all) to 3 (almost daily). Total scores range from 0-21 and are classified as follows: 0-4: "none" 5-9: "mild" 10-14: "moderate" and 15: "severe" (9). The reliability and validity of this scale on a Turkish sample was verified by Konkan et al. (10).

During the pandemic, face-to-face interviews with patients would be much riskier for those in the older age group, so we decided on phone interviews. Moreover, PHQ-9 and GAD-7 was administered online to the participants after the phone call, there might be difficulties in adapting to the questionnaire, and data reliability might decrease when they ask others to fill in the questionnaires, all of which could lead to significant data loss. Thus, all the scale questions were read one by one, and the patients' scores were recorded during the phone interviews.

\section{Statistical analysis}

IBM SPSS Statistics Version 22.0 was used to analyze all statistical data. The standard deviation for continuous data and the frequencies and percentages for categorical data were used as descriptive statistics. Normal data distribution was tested using the Kolmogorov-Smirnov test. Student's t-test was used for the continuous variables, and the chi-square test was used for the categorical variables to determine the differences between groups. The cut-off for GAD-7 and PHQ-9 was accepted as $\geq 5$. Binary logistic regression analysis was used to predict the cases with higher anxiety (GAD-7 scores $\geq 5$ ) and higher depression (PHQ scores $\geq 5$ ) levels. A value of $p<0.05$ was considered statistically significant.

\section{RESULTS}

Of the 776 patients, 463 (59.7\%) were women, and $313(37.6 \%)$ were men. The mean age was $73.4 \pm 6.6$ years and the mean education duration was $6.5 \pm 4.8$ years. Regarding marital status, $482(62.1 \%)$ of the 
participants were married, while 294 (37.9\%) were widowed/divorced/single. The sociodemographic information of the participants is given in Table 1. During the telephone interviews, 386 (49.7\%) of the 776 participants were interviewed along with their relatives.

A total of 770 participants (99.2\%) were not working before the pandemic, as 434 (55.9\%) were retired and 336 (43.3\%) were unemployed. Only a small group of patients $(n=6,0.8 \%)$ had to leave their jobs during the pandemic. In terms of living arrangements, 692 (89.2\%) of the patients lived with their spouse or spouse and children, and 84 (10.8\%) lived alone. After the pandemic, only a small group (2.4\%) changed their living environment due to isolation, and most of the patients (97.6\%) did not make any changes to their family lives.

When the curfew started, 45 (5.8\%) of the patients continued to meet their basic needs themselves, but 731 (94.2\%) needed the help of their families, friends, neighbors, or others to fulfill these needs. When the patients were asked whether their close family members supported their basic needs, $100(12.9 \%)$ reported that they did not receive any support from their family members.

During the pandemic, 43 (5.5\%) of the patients could go to outpatient visits, while 733 (94.5\%) were unable to do the same. The most common reason for not going to outpatient visits was anxiety related to contracting the COVID-19 virus ( $n=427,55.0 \%)$, followed by curfew ( $n=144,18.6 \%$ ), no need to go $(n=38,4.9 \%)$, and the inability to set an appointment $(n=28,3.6 \%)$.

As for taking their psychiatric medications, 607 (78.2\%) of the patients regularly took theirs, while 169 (21.8\%) did not. Furthermore, we found that 442 (72.8\%) of the patients who took their medicines regularly were able to procure them directly from a pharmacy without a prescription. The extension of prescriptions during the pandemic enabled them to buy and take their medications regularly.

Among the patients, 554 (72.7\%) indicated that they would like to contact their physician over the

Table 1. Socio-demographic characteristics of the patients

\begin{tabular}{|l|l|c|}
\hline Age (mean \pm SD) (years) & & $73.4 \pm 6.6$, \\
\hline Gender (n, \%) & Male & $313(37.6 \%)$ \\
\hline & Female & $463(59.7 \%)$ \\
\hline Marital Status (n, \%) & Married & $294(37.9 \%)$ \\
\hline & Widow/divorced/single & $6.5 \pm 4.8$ \\
\hline Education (years) & & $770(99.2 \%)$ \\
\hline Employment Status (n, \%) & Unemployment/retired & $6(0.8 \%)$ \\
\hline & Working before pandemic & $692(89.2 \%)$ \\
\hline
\end{tabular}

n: number of people, \%: percentage 
phone or the Internet in the event of a pandemic or extraordinary crisis. However, only seven people $(0.9 \%)$ reported that they were contacted online by their follow-up doctors during the pandemic.

\section{Psychiatric diagnoses based on hospital re- cords and the prevalence of anxiety-depressive symptoms}

The diagnoses of the patients based on hospital records were as follows: anxiety disorders: 490 (63.2\%) patients, depression: 112 (14.4\%) patients, dementia or cognitive disorders: 83 (10.7\%) patients, schizophrenia spectrum disorders: 35 (4.5\%) patients, sleep disorders: 35 (4.5\%) patients, bipolar disorder: 18 (2.3\%) patients, and adjustment disorder: $3(0.4 \%)$ patients. The mean duration of psychiatric illness in all patients was $94.9 \pm 122.4$ months (min: 6, max: 660 months). In addition, 53 (6.9\%) patients were hospitalized at least once in the psychiatric inpatient unit. Regarding patients' treatments, the average number of psychotropic drug intake was $1.08 \pm 0.71$ ( $\min : 0$, max: 4).

The most used antidepressants were escitalopram $(n=170,21.9 \%)$ and sertraline $(n=169,21.8 \%)$. The most frequently used antipsychotic drugs were quetiapine $(n=113,14.6 \%)$ and olanzapine $(n=38$, 4.9\%). In addition, 13 (1.7\%) patients were using benzodiazepine; 10 (1.3\%) were using valproic acid, lithium, or carbamazepine; and 11 (1.4\%) were using long-acting antipsychotics.

Regarding the scales, 53 (6.8\%) and 133 (17.1\%) patients had GAD-7 scores of $\geq 10$ and $\geq 5$, respectively, whereas 52 (6.7\%) and 102 (14\%) patients had PHQ-9 scores of $\geq 10$ and $\geq 5$, respectively.

When the patients' psychiatric complaints after the pandemic were evaluated, we found that 318 (41.0\%) had an increase in their psychological complaints. The most frequently reported complaint was an increase in anxiety level (23.7\%), followed by insomnia (17.0\%), and depression (13.3\%), as shown in Table 2.
In all cases, the sociodemographic and clinical features were compared according to the scores obtained from the GAD-7 and PHQ-9 scales ( $<5$ and $\geq 5$ ). Regular drug use was lower in those with high anxiety levels. Those with high depression levels were older. In addition, female sex ratio and single/ widow status were higher in those with $\mathrm{PHQ}-9 \geq 5$. Comparison of General Anxiety Disorder-7 (GAD-7) and Patient Health Questionnaire-9 (PHQ-9) scores according to socio-demographic and clinical characteristics of the patients are shown in Table 3.

Our aim was to predict the cases with higher anxiety (GAD-7 scores $\geq 5$ ) and higher depression ( $P H Q$ 9 scores $\geq 5$ ) levels. Statistically significant difference in the first line analysis $(p<0.05)$ were included as independent variables in binary logistic analysis. Variables with $p<0.05$ for higher anxiety levels (GAD-7 scores $\geq 5$ ) were "duration of psychiatric illness" $(<5$ years $=0, \geq 5$ years $=1$ ) and "regular drug use in the pandemic" (yes $=0, n o=1$ ). Variables with $p<0.05$ for high depression levels (PHQ-9 scores $\geq 5$ ) were "age," "gender" (male=0, female=1), and "marital status" (married=0, single/widow=1). Binary logistic regression analysis was performed by grouping categorical variables as 0 and 1 . Results are shown in Table 4.

\section{DISCUSSION}

Many studies have examined how different societies, age groups, and certain occupational groups, especially healthcare professionals, have been psychologically affected by the COVID-19 pandemic (11). Among elderly patients, more physiological studies than psychological research have been conducted due to the high mortality rates in this group. However, it was stated that during the pandemic, the elderly experienced problems such as not being physically together with their loved ones, decreased social activities, and loneliness, which were associated with increased mortality and morbidity (12). It was reported by Korkmaz et al. that the elderly experienced depressive complaints such as 
Table 2. Diagnosis of psychiatric illness, duration of illness and psychiatric complaints of the patients

\begin{tabular}{|l|l|c|}
\hline & & $\mathbf{n}, \%$ \\
\hline Psychiatric diagnosis & Anxiety Disorders & $490(63.2 \%)$ \\
\hline & Major depression & $112(14.4 \%)$ \\
\hline & Dementia or cognitive impairment & $83(10.7 \%)$ \\
\hline & Schizophrenia spectrum disorders & $35(4.5 \%)$ \\
\hline & Sleep disorders & $35(4.5 \%)$ \\
\hline & Bipolar disorder & $18(2.3 \%)$ \\
\hline $\begin{array}{l}\text { Duration of illness (mean } \pm \text { SD) } \\
\text { (months) }\end{array}$ & Adjustment disorder. & $3(0.4 \%)$ \\
\hline Change of psychiatric complaints & Increased & $94.9 \pm 122.4$ (min: 6 max: 660) \\
\hline & Not changed or decreased & $318(41.0 \%)$ \\
\hline Type of psychiatric complaints & Anxiety & $458(59.0 \%)$ \\
\hline & Insomnia & $184(23.7)$ \\
\hline & Depression & $132(17.0)$ \\
\hline & Irritability & $103(13.3)$ \\
\hline & Restlessness & $98(12.6)$ \\
\hline & Decreased energy & $78(10.1)$ \\
\hline & Decreased appetite & $57(7.3)$ \\
\hline & Forgetfulness & $40(5.2)$ \\
\hline & Distractibility & $31(4.0)$ \\
\hline Regular drug use & Yes & $23(3.0)$ \\
\hline & No & 607 (78.2\%) \\
\hline & & $169(21.8 \%)$ \\
\hline & & $44.4 \pm 70.9($ min:0 max:480) \\
\hline
\end{tabular}

n: number of people, \%: percentage

sleep problems, fatigue and hopelessness during the pandemic period, and the risk of depression increases as the hospital admission is prolonged (13). It is important to follow up psychological complaints in elderly patients and continue the treatment regularly if a mental disorder has developed or is present (14). In this process, for the arrangements to be made in health services, it is necessary to determine the problems related to the continuation of treatment and the increase in mental complaints of these people. To the best of our knowledge, our study is the first to evaluate how geriatric psychiatric patients achieve treatment compliance, how they receive social support, and whether they are affected mentally during this period. This is a cross-sectional study that retrospectively examines the system records and evaluates each patient's current condition via phone calls.

Furthermore, elderly individuals have a greater risk for acute respiratory distress syndrome and higher mortality rates due to COVID-19 (15). Compared to younger individuals, older adults may be cognitively impaired and more sensitive to stress, which could lead to psychiatric disorders (16). This population group also experiences poor social and family support, which in turn, may increase loneli- 
Table 3. Comparison of General Anxiety Disorder-7 (GAD-7) and Patient Health Questionnaire-9 (PHQ-9) scores according to socio-demographic and clinical characteristics of the patients

\begin{tabular}{|c|c|c|c|c|c|c|c|c|c|}
\hline & & \multicolumn{4}{|c|}{ GAD-7 } & \multicolumn{4}{|c|}{ PHQ-9 } \\
\hline & & $<5$ & $\geq 5$ & $x^{2} / t^{\star}$ & $p$ & $<5$ & $\geq 5$ & $x^{2} / t^{*}$ & $p$ \\
\hline $\begin{array}{l}\text { Age }(\text { mean } \pm S D) \\
\text { (years) }\end{array}$ & & $73.5 \pm 6.7$ & $73.1 \pm 6.3$ & 0.717 & 0.477 & $73.2 \pm 6.5$ & $74.8 \pm 7.0$ & -2.327 & 0.020 \\
\hline \multirow[t]{2}{*}{ Gender (n, \%) } & Female & $382(82.5)$ & $81(17.5)$ & 0.102 & 0.749 & $386(83.4)$ & $77(16.6)$ & 6.350 & 0.012 \\
\hline & Male & $261(83.4$ & $52(16.6)$ & & & $281(89.8)$ & $32(10.2)$ & & \\
\hline \multirow[t]{2}{*}{ Marital status (n, \%) } & Married & 404(83.8 & $78(16.2)$ & 0.820 & 0.365 & $425(88.2)$ & $57(11.8)$ & 5.196 & 0.023 \\
\hline & Single/widow & $239(81.3)$ & $55(18.7)$ & & & $242(82.3)$ & $52(17.7)$ & & \\
\hline \multirow[t]{2}{*}{$\begin{array}{l}\text { Education } \\
(n, \%)\end{array}$} & $\begin{array}{l}\leq \text { Secondary school } \\
(n: 508)\end{array}$ & $418(82.3)$ & $90(17.7)$ & 0.345 & 0.557 & $430(84.6)$ & $78(15.4$ & 2.084 & 0.149 \\
\hline & $\begin{array}{l}\geq \text { High school (n: } \\
268)\end{array}$ & $225(84.0)$ & $43(16.0)$ & & & $237(88.4)$ & $31(11.6)$ & & \\
\hline \multirow{2}{*}{$\begin{array}{l}\text { Duration of psychiat- } \\
\text { ric illness }(n, \%)\end{array}$} & $<5$ years & $420(85.9)$ & $69(14.1)$ & 8.540 & 0.003 & $426(87.1)$ & $63(12.9)$ & 1.481 & 0.224 \\
\hline & $\geq 5$ years & $223(77.7)$ & $64(22.3)$ & & & $241(84.0)$ & $46(16.0)$ & & \\
\hline \multirow{2}{*}{$\begin{array}{l}\text { Duration of regular } \\
\text { drug use }(n, \%)\end{array}$} & $<6$ months & $146(78.5)$ & $40(21.5)$ & 3.284 & 0.070 & 155(83.3) & $31(16.7)$ & 1.397 & $0.238)$ \\
\hline & $\geq 6$ months & $497(84.2)$ & 93(15.8) & & & $512(86.8)$ & $78(13.2)$ & & \\
\hline \multirow{2}{*}{$\begin{array}{l}\text { Regular drug use in } \\
\text { pandemic }(n, \%)\end{array}$} & Yes & $512(84.3)$ & 95(15.7) & 4.348 & 0.037 & $523(86.2)$ & $84(13.8)$ & 0.100 & 0.752 \\
\hline & No & $131(77.5)$ & $38(22.5)$ & & & $144(85.2$ & $25(14.8)$ & & \\
\hline
\end{tabular}

n: number of people, \%: percentage, ${ }^{\star}$ Chi-square test was used to compare categorical variables and Student's t-test was used to compare continuous variables

Table 4. Binary Logistic regression of General Anxiety Disorder-7 (GAD-7) $\geq 5$ and Patient Health Questionnaire-9 (PHQ-9) $\geq 5$

\begin{tabular}{|c|c|c|c|c|c|c|}
\hline Scale & Variables & $B$ & S.E. & Wald & $\begin{array}{l}\text { p-val- } \\
\text { ue }\end{array}$ & $\operatorname{Exp}(B)$ \\
\hline \multirow[t]{3}{*}{$\begin{array}{ll} & \mathrm{PHO}- \\
9^{a} & \\
\end{array}$} & Age & 0.032 & 0.015 & 4.88 & 0.027 & 1.033 \\
\hline & Gender (male) & 0.545 & 0.225 & 5.858 & 0.016 & 1.725 \\
\hline & Constant & -4.561 & 1.105 & 17.041 & $<0.001$ & 0.01 \\
\hline \multirow[t]{3}{*}{7 GAD- } & Regular drug use (yes) & 0.552 & 0.22 & 6.289 & 0.012 & 0.576 \\
\hline & Duration of psychiatric illness ( $<5$ years) & 0.628 & 0.196 & 10.292 & 0.001 & 1.875 \\
\hline & Constant & -1.418 & 0.196 & 52.412 & $<0.001$ & 0.242 \\
\hline
\end{tabular}

aPHQ-9: Omnibus Test of Model Coefficients: $\chi 2=11.278, p=0.004$, Adjusted R2: 0,026. ${ }^{b}$ GAD-7: Omnibus Test of Model Coefficients: $\chi^{2}=14.354, p=0.001$, Adjusted R2:0,031 
ness, despair, and hopelessness, all of which are determinants of suicide. Unfortunately, due to the limited mental health services available during a pandemic, the elderly may have problems undergoing psychiatric examination. Another important factor to consider is the fact that the elderly may not have access to adequate treatment due to the difficulties they experience in Internet and smartphone use and access (17).

In our study, a large proportion of patients (94.2\%) needed the help of their families, friends, neighbors, or others to fulfill their basic needs. $12.9 \%$ stated that they did not receive any support from their family members. This rate could not be compared with the literature data due to the lack of a similar study.

In treating psychiatric diseases, patients need to maintain regular treatment and outpatient visits. Although $94.5 \%$ of our patients could not go to outpatient visits due to COVID-19 anxiety and curfew, $78.2 \%$ were able to continue their treatment. Moreover, the vast majority were able to obtain their medication directly from the pharmacy without the need for updated prescriptions.

In the first large-scale nationwide study on psychological distress in the general population during the early stages of the COVID-19 pandemic in China, a study found that almost $35 \%$ of the participants experienced psychological distress (18). In particular, female participants in that study showed greater psychological distress, were more vulnerable to stress, and were more likely to develop trauma-related disorders than male participants. Furthermore, it has been demonstrated that people in the 18-30 and over 60 age groups have higher psychological distress measurement values compared with those in other age groups. Meng et al. examined a Chinese sample of people over 60 years old using GAD-7 and PHQ-9 and found that 37.1\% reacted emotionally in the form of anxiety or depressive symptom. Furthermore, they found a gender difference in that women had higher depression and anx- iety levels via their PHQ-9 and GAD-7 scores than male participants (19).

In the current study, $41.0 \%$ of our patients stated that their psychiatric complaints increased. The most frequently stated complaints were anxiety (23.7\%), insomnia (17\%), and depression (13.3\%). However, we found no gender difference in the levels of anxiety reported in both genders. Nevertheless, the fact that women's depression scores are higher than men's is consistent with previous studies, which reported that women have lower stress sensitivity and higher psychological exposure. In our study, the absence of gender difference in anxiety levels may be attributed to the lack of information on the patients' anxiety and depressive levels before the pandemic and the limitation of employing a cross-sectional evaluation method.

A study conducted in the early period of the pandemic in China found that $28.8 \%$ and $6.5 \%$ of the sample reported moderate-severe anxiety and moderate-severe depression symptoms, respectively (20). Another Chinese study, which was conducted using an online questionnaire survey, reported the anxiety, somatization, and insomnia prevalence rates of $33.0 \%, 7.6 \%$, and $24.5 \%$, respectively (21). In a different Chinese study that evaluated 500 people via an online survey, results revealed that 19\% of the respondents had depression (PHQ-9 score of $\geq 10$ ), and $14 \%$ had anxiety (GAD score of $\geq 10$ ) (22). In our study, a PHQ-9 score of $\geq 10$ was found in $6.7 \%$ of the patients and a GAD-7 score of $\geq 10$ was found in $6.8 \%$. Although $41.0 \%$ of our study participants reported that their psychiatric complaints increased, the rates were low when an evaluation was made according to the scale cut-off scores. This suggests that a significant number of patients have subthreshold psychiatric complaints. Hence, despite the fact that the values are below the cut-off scores, the persistence of subthreshold symptoms is an important issue that should be considered in psychiatric treatment. Therapeutic consultations with patients reporting psychiatric complaints that 
are not yet at the disease level can improve the latter's quality of life and are, therefore, highly recommended (23). Our study's low rates may also be attributed to the vast majority of patients continuing their recommended treatment at the final outpatient visit despite the pandemic.

When the anxiety scores were examined in our study, we found that they were higher in those who had been ill for more than five years. Thus, this finding is compatible with the chronic course and recurrent nature of anxiety disorders (24). In previous studies, the discontinuation of antidepressant drugs has been defined as a predictor of the recurrence of anxiety disorders (25). In our study, we found that GAD-7 scores of $\geq 5$ were statistically significantly higher in those who did not regularly take medications during the pandemic and had a disease duration of five years or more. Upon further analysis, we found that these two variables served as predictors of the patient group with GAD-7 anxiety scores of $\geq 5$.

The literature on major recurrent depression risk factors is not entirely consistent. Suspected risk factors include the number of current smokers, female gender, and being single. In comparison, the literature is fairly consistent regarding the importance of a history of depression as a risk for future episodes $(26,27)$.

Furthermore, after examining the depression scores, we found that those in the group with a PHQ-9 score of $\geq 5$ were female, had a statistically significant higher age, and were single or widowed. However, upon further examination, we found that this difference was not related at all to being female and having an increased age. This may be because the number of recurrent episodes is high, and the duration of illness is longer, in which the disease may have already turned into a chronic state.

Furthermore, $72.7 \%$ of the patients stated that they would be satisfied with having Internet or telephone communication when future situations would make it difficult for them to reach a doctor. Thus, it may be essential to design and establish easily accessible telepsychiatry applications that can be used by geriatric and psychiatric patients in similar situations in the future.

In terms of limitations, as mentioned earlier, our study evaluated patients cross-sectionally. Conducting a follow-up study by determining the severity of psychological symptoms using clinical scales before, during, and after the pandemic may guide future studies in determining the pandemic's psychological effects on the geriatric population.

Moreover, as we aimed to evaluate the psychological effects of the pandemic on geriatric psychiatric patients and their treatment-social adaptation in our study, only patients with follow-up schedules in our clinic were included. Therefore, the lack of comparison with the geriatric population without psychiatric comorbidity is a limitation, and comparative studies are recommended in future studies.

Finally, the scales applied in our study were recorded according to the information obtained via phone calls. This method was chosen because physically interviewing a geriatric patient sample during a pandemic may put them at risk. Hence, the use of online video interviews using telepsychiatry applications is recommended in future studies. This was a result of our study data, which patients also wanted. We believe that our findings, based on data obtained from interviews with patients and their relatives, will provide insights into the management of psychiatric treatments for people aged 65 and above during extraordinary situations, such as pandemics.

Funding: The researchers covered the expenses of this research. No company support or scholarship has been received.

Conflict of interest: All authors report no competing interests.

Acknowledgments: We wish to thank all the study participants for their cooperation. 


\section{REFERENCES}

1. Novel CPERE. The epidemiological characteristics of an outbreak of 2019 novel coronavirus diseases (COVID-19) in China. Zhonghua liu xing bing xue za zhi 2020;41(2):145-51. (PMID:32064853)

2. Liu K, Chen Y, Lin R, Han K. Clinical features of COVID-19 in elderly patients: A comparison with young and middle-aged patients. J Infect 2020; 80(6): e14-e18. (PMID:32171866)

3. Mehra A, Rani S, Sahoo S, et al. A crisis for elderly with mental disorders: Relapse of symptoms due to heightened anxiety due to COVID-19. Asian J Psychiatr 2020; 51:102114. (PMID:32334406)

4. Salari N, Hosseinian-Far A, Jalali R, et al. Prevalence of stress, anxiety, depression among the general population during the COVID-19 pandemic: a systematic review and meta-analysis. Glob Health 2020;16(1):1-11. (PMID:32631403)

5. Hacimusalar Y, Kahve AC, Yasar AB, Aydin MS. Anxiety and hopelessness levels in COVID-19 pandemic: A comparative study of healthcare professionals and other community sample in Turkey. J Psychiatr Res 2020;129:181-88. (PMID:32758711)

6. Pfefferbaum B, North CS. Mental health and the Covid-19 pandemic. NEJM 2020; 383(6), 510-12. (PMID:32283003)

7. Kroenke K, Spitzer RL, Williams JB. The PHQ-9: validity of a brief depression severity measure. J Gen Intern Med 2001;16(9):606-13. (PMID:11556941)

8. Sari YE, Kokoglu B, Balcioglu H, Bilge U, Colak E, Unluoglu I. Turkish reliability of the patient health questionnaire-9. Biomed Res 2016; 460-2. (ISSN: 0970-938X)

9. Spitzer RL, Kroenke K, Williams JB, Löwe B. A brief measure for assessing generalized anxiety disorder: the GAD-7. Arch Intern Med 2006;166(10):1092-97. (PMID:16717171)

10. 1Konkan R, Senormancı O, Güçlü $O$, Aydin E, Sungur MZ. Validity and Reliability Study for the Turkish Adaptation of the Generalized Anxiety Disorder-7. Arch Neuropsychiatry 2013;50(1):53-58. (DOI: 10.4274/ npa.y6308)

11. Ahmed MZ, Ahmed O, Aibao Z, Hanbin S, Siyu L, Ahmad A. Epidemic of COVID-19 in China and associated Psychological Problems. Asian J Psychiatr 2020:102092. (PMID:32315963)

12. Roy J, Jain R, Golamari R, Vunnam R, et al. COVID-19 in the geriatric population. Int J Geriatr Psychiatry 2020; 35(12): 1437-1441. (DOI: 10.1002/gps.5389)

13. 1Yurumez Korkmaz B, Gemci E, Cosarderelioglu C, et al. Attitudes of a geriatric population towards risks about COVID-19 pandemic: in the context of anxiety and depression. Psychogeriatr 2021;21:730-737. (DOI: 10.1111/psyg.12731)

14. Mackolil J., Mackolil, J. Why is mental health of the geriatric population at a higher risk during the COVID-19 pandemic? Asian J Psychiatr 2020; 54:102401. (PMID: 33271699)

15. Wang L, He W, Yu X, et al. Coronavirus disease 2019 in elderly patients: Characteristics and prognostic factors based on 4-week follow-up. J Infect 2020;80(6), 639-45. (PMID:32240670)

16. Lloyd-Sherlock PG, Kalache A, McKee M, et al. WHO must prioritize the needs of older people in its response to the covid-19 pandemic. BMJ 2020;368. (PMID:32205399)

17. Yang Y, Li W, Zhang Q, Zhang L, Cheung T, Xiang Y-T. Mental health services for older adults in China during the COVID-19 outbreak. The Lancet Psychiatry 2020;7(4):e19. (PMID: 32085843)

18. Qiu J, Shen B, Zhao M, Wang Z, Xie B, Xu Y. A nationwide survey of psychological distress among Chinese people in the COVID-19 epidemic: implications and policy recommendations. Gen Psychiatr 2020;33(2). (PMID:32215365)

19. Meng H, Xu Y, Dai J, Zhang Y, Liu B, Yang H. Analyze the psychological impact of COVID-19 among the elderly population in China and make corresponding suggestions. Psychiatry Res 2020;289:112983. (PMID:32388175)

20. Wang C, Pan R, Wan X, et al. Immediate psychological responses and associated factors during the initial stage of the 2019 coronavirus disease (COVID-19) epidemic among the general population in China. Int J Environ Res Public Health 2020;17(5):1729. (PMID:32155789)

21. Huang $Y$, Wang $Y$, Zeng $L$, et al. Prevalence and Correlation of Anxiety, Insomnia and Somatic Symptoms in a Chinese Population During the COVID-19 Epidemic. Front Psychiatry 2020;11:894. (PMID:33005165)

22. Choi EPH, Hui BPH, Wan EYF. Depression and anxiety in Hong Kong during COVID-19. Int J Environ Res Public Health 2020;17(10):3740. (PMID:32466251)

23. 23. Kasckow J, Karp J, Whyte E, et al. Subsyndro- 
mal depression and anxiety in older adults: health related, functional, cognitive and diagnostic implications. J Psychiatr Res 2013;47(5):599-603. (PMID: 23414701)

24. Bruce SE, Yonkers KA, Otto MW, et al. Influence of psychiatric comorbidity on recovery and recurrence in generalized anxiety disorder, social phobia, and panic disorder: a 12-year prospective study. Am J Psychiatry 2005;162(6):1179-87. (PMID: 15930067)

25. Donovan MR, Glue P, Kolluri S, Emir B. Comparative efficacy of antidepressants in preventing relapse in anxiety disorders - a meta-analysis. J Affect Disord 2010;123(1-3):9-16. (PMID:19616306)

26. Bulloch A, Williams J, Lavorato D, Patten S. Recurrence of major depressive episodes is strongly dependent on the number of previous episodes. Depress Anxiety 2014;31(1):72-6. (PMID:24038800)

27. Hardeveld F, Spijker J, De Graaf R, Nolen W, Beekman A. Prevalence and predictors of recurrence of major depressive disorder in the adult population. Acta Psychiatr Scand 2010;122(3):184-91. (PMID:20003092) 